\title{
Implementación del sistema de Costos por Ordenes Especificas en la Empresa de Transportes San Martin de Porres del Distrito de Huacho
}

\author{
Implementation of the system of charges for orders specific in the company of \\ transport San Martin de Porres of the District of Huacho
}

Delfina Corali de los Santos Ronceros ${ }^{1}$, Miguel Angel Suarez Almeira ${ }^{1}$, Raúl Daniel Ramos Ramirez ${ }^{1}$ Pablo Fernando Vásquez Morante ${ }^{1}$, Marino Valladares Celi $^{1}$

\section{RESUMEN}

Objetivo. Determinar la relación que existe entre la implementación del sistema de costos y los costos por órdenes especificas en la empresa de transportes San Martin de Porres del Distrito de huacho tomándose como dimensiones: Control, Elaboración, Informe. Material y Métodos. La población de estudio fueron 50 personas de las diferentes Áreas de la empresa mediante el muestreo probabilístico, se utilizó el instrumento de medición de actitudes de escala de Likert. La confiabilidad de los instrumentos fue validado mediante el coeficiente alfa de Cronbach (0,945 y 0.960). La Prueba de Rho de Spearman. Resultados. Se muestran que el mayor porcentaje $(38,00 \%)$ de personas responden definitivamente si, están de acuerdo con la implementación de un sistema de costos, en cuanto al sistema de costos por órdenes específicas reporta un porcentaje mayor de 42,0\% con una opinión definitivamente si, lo que significa que están de acuerdo. Conclusión. Los resultados obtenidos demuestran que existe una correlación positiva significativa moderada entre la implementación del sistema de costos y el sistema de costos por órdenes especificas $(R=0,540 ; p=0.00<0.05)$.

Palabras clave: Control, Elaboración, Informes, Costos de los materiales, costos de la mano de obra.

\section{ABSTRACT}

Objective. Determine the relationship between the implementation of the system's costs and costs for orders specific in the transport company San Martin de Porres district of huacho taking as dimensions: Control, processing, report. Material and methods. The study population were 50 people from different Areas of the company using the probabilistic sampling, we used the Likert scale attitude measurement instrument. The reliability of the instruments was validated using Cronbach's alpha coefficient $(0,945$ and 0.960$)$. Playboy test of Rho. Results. Its show that the highest percentage (38.00\%) of people respond, if they are in accordance with the implementation of a system of cost, in terms of the system's costs for specific orders reported one higher percentage of $42,0 \%$ with an opinion definitely Yes, which means that they are in agreement. Conclusion. The results obtained show that there is a significant positive correlation moderate between the cost system implementation and system costs by orders specific $(R=0,540 ; p=0.00<0.05)$.

Keywords: Control, processing, reports, cost of materials, labor costs.

\footnotetext{
${ }^{1}$ Docente. Facultad de Ciencias Económicas, Contables y Financieras. Universidad Nacional José Faustino Sánchez Carrión. Email: coralidlsr@hotmail.com
} 


\section{INTRODUCCIÓN}

En la provincia de Huaura y principalmente en el Distrito de Huacho el mercado del transporte interprovincial de pasajeros está constituido por aproximadamente 5 personas jurídicas, de las cuales el mayor parque automotor lo conforman cerca de 3 medianas entidades, y existen cerca de 2 pequeñas empresas las cuales ofrecen la participación del $40 \%$ del mercado total ya que estas son las que cuentan con un buen número de demanda de pasajeros.

Estas empresas de transporte especial se caracterizan porque comparten problemáticas endógenas y exógenas. Las endógenas tienen que ver con aspectos o fenómenos como la falta de investigación en el sector, ausencia de personal debidamente calificado para realizar cada una de las tareas de la empresa, falta de inversión en propiedad intelectual, tales factores a nivel interno y a nivel exógeno se producen por el incremento descontrolado de empresas en el mercado, la falta de regulación y principalmente la competencia desleal lo cual afecta el desarrollo de una metodología unificada sobre la cual se pueda calcular y definir el costo del servicio.

La falta de una estructura de costos definida ocasiona que los precios se transformen en inestables y tiendan a decrecer año tras año. Si bien las disminuciones obedecen a factores tales como mayores comisiones hacia los clientes y menores márgenes de utilidad para la empresa.

En este sentido, la calidad se constituye en las empresas de transporte especial en uno de los medios fundamentales para atraer y mantener los clientes, pero es indispensable para lograr el aseguramiento de dicha calidad tener una estructura de costos debidamente definida, y es por esta razón, que las empresas no han logrado mantener una calidad total en el servicio, representada principalmente en el cumplimiento del servicio hacia el cliente final así como del contratante del servicio, con excelentes vehículos y la adecuada atención al cliente.

Aquí buscaban determinar el costo en el que incurre una empresa de carrocerías teniendo como elementos principales los materiales: mano de obra, el combustible, los aceites, y el desgaste de las máquinas y equipos de trabajo.

Querían dar solución a un problema que aqueja a la gran mayoría de las pequeñas empresas; la determinación de sus costos, se pudo establecer que estos entes operan si en realidad obtienen utilidad o no, por lo cual la investigación plantea que el aplicar un sistema de costos por órdenes especificas si podría mejorar la rentabilidad de la empresa en estudio.

Según B. Morton, L. Jacobsen y D. Jiménez (1988) En el costeo por órdenes, órdenes la tendencia actual es a automatizar las funciones de recolección y captura de datos que se requieren para apoyar al sistema de contabilidad.

J. Garcia (2001) expresa: El sistema de contabilidad de costos por órdenes específicas, conocido también como sistema de costos por órdenes de producción, por lotes, por pedido u órdenes de trabajo, es un sistema de acumulación de costos de acuerdo a las especificaciones de los clientes o en algunos casos para ser llevados al almacén de productos terminados, en el cual los costos que intervienen en el proceso de producción de una cantidad específica o definida de productos se acumulan o recopilan sucesivamente por sus elementos (materiales directos, mano de obra directa y costos indirectos), los cuales se cargan a una orden de trabajo, fabricación o producción, sin importar los periodos de tiempo que implica.

El objetivo de la Investigación es determinar la relación que existe entre los sistemas de costos y el sistema de costos por órdenes específicas de la empresa de transporte de pasajeros del distrito de Huacho.

La presente investigación beneficia a la rama de transporte terrestre automotor de pasajeros. Esta modalidad de servicio va dirigida principalmente a las personas, empresas y colegios quienes solicitan especificaciones de tipo técnico según sus necesidades. La dinámica general y la regulación establecida exigen que estos procesos se realicen a través de intermediarios, es decir; cliente-empresavehículo.

El trabajo de investigación plantea la siguiente hipótesis La implementación de un sistema de costos para la empresa Transportes San Martín de Porres del distrito de Huacho basada en el sistema de costos por órdenes específicas determinara si este sistema contribuye al mejoramiento de la eficacia y eficiencia en la situación particular de la empresa. 


\section{MATERIAL Y MÉTODOS}

Para la ejecución de la investigación se utilizó el tipo de estudio correlacionar transversal. El área de estudio considerado fue la Empresa de Transporte de pasajeros San Martin de Porres S.A del distrito de Huacho. La población de estudio fueron 50 personas, seleccionadas mediante muestreo por conveniencia con un tamaño muestral de 23 distribuidos proporcionalmente al tamaño de la población. El instrumento de medición de actitudes estuvo basado en la escala tipo Likert. El procesamiento de datos se realizo utilizando el Software Estadístico Statistical Packege Social Sciencies (SPSS) versión 22 y el software de hoja de cálculo Microsoft Office Excel 2010.

Para el procesamiento del cuestionario se utilizó la escala Likert, cuya puntuación por ítems corresponde a las respuestas dadas por 6 ejecutivos, personal administrativo 10, y 7 trabajadores de la diversas áreas der la empresa, asignándose el siguiente puntaje, Nunca $=1$; Pocas Veces $=2$; a veces $=3$; frecuentemente $=4$; siempre $=5$.

Para determinar la confiabilidad del instrumento se calculó el Alpha de Cronbach. El instrumento mostró 20 ítems, distribuidos en 11 ítems para la variable independiente y de 9 ítems para la variable dependiente, considerando a los 23 (trabajadores) encuestados: Para el instrumento de la variable independiente y dependiente se obtuvo un coeficiente de confiabilidad Alpha de Cronbach de 0.945 y 0.960 respectivamente, lo que puede interpretarse como un instrumento de alta confiabilidad.

Los datos se recodificaron para determinar algunas variables para las pruebas de independencia con Chi cuadrada $\left(X^{2}\right)$.Para la realización de la prueba y con la finalidad de cumplir con el mínimo de 5 observaciones para las celdas, se agruparon las respuestas de: Definitivamente si, probablemente si, no estoy seguro, probablemente no y definitivamente no. Se empleó la prueba de Chi cuadrado y la Prueba Rho de Spearman.

Las hipótesis en estudio fueron:

Los mecanismos de medida bajo las cuales se efectuara el costo de servicio del transporte, a partir del sistema de órdenes específicas generaran un marco de referencia bajo el cual se puede realizar el costo.

\section{Planteamiento de las hipótesis estadísticas al nivel de significación del $\alpha=5 \%$ ० $1 \%$}

$\boldsymbol{H}_{\mathrm{o}}: \boldsymbol{\rho}_{\mathrm{rs}}=\boldsymbol{0}$ : Los mecanismos de medida bajo las cuales se efectuara el costo de servicio del transporte, a partir del sistema de órdenes específicas no generaran un marco de referencia bajo el cual se puede realizar el costo.

$\boldsymbol{H}_{1}: \boldsymbol{\rho}_{\mathrm{rs}} \neq \boldsymbol{0}$ : Los mecanismos de medida bajo las cuales se efectuara el costo de servicio del transporte, a partir del sistema de órdenes específicas generaran un marco de referencia bajo el cual se puede realizar el costo.

\section{RESULTADOS}

Con el fin de comprobar la hipótesis nula se aplicó la prueba $\mathrm{chi}^{2}$ dando por resultado que se rechaza la hipótesis general nula con un $\alpha=0.000$, aceptando que existe relación directa entre la implementación del sistema de costos y el sistema de costos por órdenes especificas en la empresa de transportes San Martin de Porres S:A del distrito de Huacho.

En cuanto a la primera hipótesis específica al obtenerse un $\alpha=0.000$ se rechazó la hipótesis nula, aceptando que existe relación directa entre las variables Control de costos del servicio y el Costo de los Materiales en la empresa de transportes san Martin de Porres S.A del distrito de Huacho.

Con respecto a la segunda hipótesis específica al obtenerse un $\alpha=0.000$ se rechaza la hipótesis nula aceptando que existe relación directa entre las variables; organización de una base de datos de vehículos y el desarrollo del sistema de costos por órdenes específicas de la empresa de transporte San Martin de Porres S.A del distrito de Huacho.

Con respecto a la tercera hipótesis específica al obtenerse un $\alpha=0.000$ se rechaza la hipótesis nula aceptando que existe relación directa entre las variables La formalización y evaluación del costo, mediante el impacto del sistema de costos por órdenes específicas en la Empresa de Transportes San Martin de Porres S.A del Distrito de Huacho. 
Tabla 1. Valores obtenidos de la prueba Chi Cuadrado $\left(x^{2}\right)$ evaluada

\begin{tabular}{|c|c|c|c|}
\hline \multirow{2}{*}{\multicolumn{2}{|c|}{ Control de los costos de servicio }} & \multicolumn{2}{|c|}{ Costos de materiales } \\
\hline & & $\begin{array}{l}\text { Costos de las } \\
\text { Ilantas. }\end{array}$ & $\begin{array}{c}\text { Costo de los materiales } \\
\text { de consumo de } \\
\text { combustible por unidad }\end{array}$ \\
\hline $\begin{array}{l}\text { Planificación de } \\
\text { resultados }\end{array}$ & $\begin{array}{l}\text { Coeficiente de } \\
\text { correlación Rho } \\
\text { de Spearman } \\
\text { Sig. (bilateral) } \\
\text { n }\end{array}$ & $\begin{array}{r}r_{\mathrm{sp}}=0,462^{*} \\
\mathrm{p}=0,023 \\
24\end{array}$ & $\begin{array}{r}r_{s p}=0,123 \\
p=0,567 \\
24\end{array}$ \\
\hline $\begin{array}{l}\text { Planificación de } \\
\text { costos de materiales }\end{array}$ & $\begin{array}{l}\text { Coeficiente de } \\
\text { correlación Rho } \\
\text { de Spearman } \\
\text { Sig. (bilateral) } \\
\text { n }\end{array}$ & $\begin{array}{r}r_{s p}=0,507^{*} \\
p=0,011 \\
24\end{array}$ & $\begin{array}{r}r_{s p}=0,424^{\star} \\
p=0,039 \\
24\end{array}$ \\
\hline Demanda de clientes & $\begin{array}{l}\text { Coeficiente de } \\
\text { correlación Rho } \\
\text { de Spearman } \\
\text { Sig. (bilateral) } \\
\text { n }\end{array}$ & $\begin{array}{r}r_{s p}=0,162^{*} \\
p=0,451 \\
24\end{array}$ & $\begin{array}{r}r_{s p}=0,237 \\
p=0,265 \\
24\end{array}$ \\
\hline $\begin{array}{l}\text { Costos de materiales } \\
\text { por vehiculo }\end{array}$ & $\begin{array}{l}\text { Coeficiente de } \\
\text { correlación Rho } \\
\text { de Spearman } \\
\text { Sig. (bilateral) } \\
\text { N }\end{array}$ & $r_{\mathrm{sp}}=0,463^{*}$ & $\begin{array}{r}r_{\mathrm{sp}}=0,480^{*} \\
p=0,018 \\
24\end{array}$ \\
\hline $\begin{array}{l}\text { Identificación } \\
\text { del sistema }\end{array}$ & $\begin{array}{l}\text { Coeficiente de } \\
\text { correlación Rho } \\
\text { de Spearman } \\
\text { Sig. (bilateral) } \\
\text { N }\end{array}$ & $\begin{array}{r}r_{s p}=0,339 \\
p=0,105 \\
24\end{array}$ & $\begin{array}{r}r_{\mathrm{sp}}=0,396 \\
p=0,055 \\
24\end{array}$ \\
\hline
\end{tabular}

Fuente: Elaboración Propia

si $p<\alpha=0,05$ se rechaza la hipótesis $H_{0}$, siendo la correlación significativa entre los indicadores control de los costos y costos de los materiales de las variables de la investigación.

\section{Interpretación}

\section{Correlaciones significativas, siendo $p<\alpha=0,0500,01$}

Planificación de resultados y Costo de las llantas.

Planificación de costos de materiales y Costo de las llantas.

Planificación de costos de materiales y Costo de los materiales de consumo de combustible por unidad.

Costos de materiales por vehículo y Costo de las llantas.

Costos de materiales por vehículo y Costo de los materiales de consumo de combustible por unidad.

\section{Correlaciones no significativas, siendo $p>\alpha=0,05$}

Planificación de resultados y Costo de los materiales de consumo de combustible por unidad.

Demanda de clientes y Costo de las Ilantas.

Demanda de clientes y Costo de los materiales de consumo de combustible por unidad.

Identificación del sistema y Costo de las llantas.
Identificación del sistema y Identificación del sistema y Costo de las llantas.

\section{Segunda hipótesis especifica}

La organización de una base de datos de vehículos sobre la cual se pueda desarrollar el costo por órdenes específicas mediante diálogos, valoraciones y encuestas a la empresa existente en el sector servirá para tener La base de cálculo de las variables definidas para el costo de servicio.

\section{Planteamiento de las hipótesis estadísticas al nivel de significación del $\alpha=5 \%$ o $1 \%$}

$\boldsymbol{H}_{o}: \boldsymbol{\rho}_{r s}=\mathbf{0}:$ La organización de una base de datos de vehículos sobre la cual se pueda desarrollar el costo por órdenes específicas mediante diálogos, valoraciones y encuestas a la empresa existente en el sector no servirá para tener La base de cálculo de las variables definidas para el costo de

$\boldsymbol{H}_{1}: \boldsymbol{\rho}_{\mathrm{rs}} \neq \boldsymbol{0}:$ La organización de una base de datos de vehículos sobre la cual se pueda desarrollar el costo por órdenes específicas mediante diálogos, valoraciones y encuestas a la empresa existente en el sector servirá para tener La base de cálculo de las variables definidas para el costo de servicio.

Tabla 2. Valores obtenidos de la prueba de Chi Cuadrado $\left(X^{2}\right)$ evaluada.

\begin{tabular}{|c|c|c|c|c|}
\hline \multirow[b]{2}{*}{ Elaboración de } & \multirow[b]{2}{*}{ Costos } & \multicolumn{3}{|c|}{ Costos de mano de obra } \\
\hline & & $\begin{array}{l}\text { Costos de mano } \\
\text { de obra de } \\
\text { choferes por } \\
\text { semana }\end{array}$ & $\begin{array}{l}\text { Costos de mano } \\
\text { de obra de } \\
\text { choferes por } \\
\text { unidad }\end{array}$ & $\begin{array}{c}\text { Costos de mano } \\
\text { de obra de las } \\
\text { terramozas }\end{array}$ \\
\hline $\begin{array}{l}\text { Costo por unidad } \\
\text { de transporte }\end{array}$ & $\begin{array}{l}\text { Coeficiente de } \\
\text { correlación Rho } \\
\text { de Spearman } \\
\text { Sig. (bilateral) } \\
n\end{array}$ & $\begin{array}{r}p=0,004 \\
24\end{array}$ & $\begin{array}{r}p=0,503 \\
24\end{array}$ & $\begin{array}{r}r_{s p}=0,731^{* *} \\
p=0,000 \\
24\end{array}$ \\
\hline $\begin{array}{l}\text { Costos de } \\
\text { materiales por } \\
\text { vehículo }\end{array}$ & $\begin{array}{l}\text { Coeficiente de } \\
\text { correlación Rho } \\
\text { de Spearman } \\
\text { Sig. (bilateral) } \\
\text { n }\end{array}$ & $\begin{array}{r}r_{s p}=0-, 009 \\
p=0,966 \\
24\end{array}$ & $\begin{array}{r}r_{\text {sp }}=0,045 \\
p=0,834 \\
24\end{array}$ & $\begin{array}{r}r_{s p}=0,367 \\
p=0,077 \\
24\end{array}$ \\
\hline $\begin{array}{l}\text { Elaboración del } \\
\text { costo de la mano } \\
\text { de obra }\end{array}$ & $\begin{array}{l}\text { Coeficiente de } \\
\text { correlación Rho } \\
\text { de Spearman } \\
\text { Sig. (bilateral) } \\
n\end{array}$ & $\begin{array}{r}p=0,162 \\
24\end{array}$ & $\begin{array}{r}r_{\text {sp }}=0,248 \\
p=0,242 \\
24\end{array}$ & $\begin{array}{r}r_{s p}=0,461^{*} \\
p=0,023 \\
24\end{array}$ \\
\hline $\begin{array}{l}\text { Costos indirectos } \\
\text { por unidad }\end{array}$ & $\begin{array}{l}\text { Coeficiente de } \\
\text { correlación Rho } \\
\text { de Spearman } \\
\text { Sig. (bilateral) } \\
n\end{array}$ & $r_{\mathrm{sp}}=0,269$ & $\begin{array}{r}p=0,757 \\
24\end{array}$ & $\mathrm{r}_{\mathrm{sp}}=0,500^{*}$ \\
\hline
\end{tabular}

Fuente: Elaboración Propia 
Si $p<\alpha=0,05$ se rechaza la hipótesis $H_{0}$, siendo la correlación significativa $\left(^{*}\right)$ entre los indicadores elaboración de datos y costos de mano de obra de las variables de la investigación

Si $p<\alpha=0,05$ se rechaza la hipótesis $H_{0}$, siendo la correlación altamente significativa $\left({ }^{* *}\right)$ entre los indicadores elaboración de datos y costos de mano de obra de las variables de la investigación.

\section{Interpretación}

Correlaciones significativas, siendo $p<\alpha=0,0500,01$

Costo por unidad de transporte y costo de mano de obra de terramozas

Costo por unidad de transporte y costo de mano de obra de choferes por semana

Costos de materiales por vehículo y costos de mano de obra de terramozas

Elaboración del costo de la mano de obra y el costo de mano de obra de terramozas

Costos indirectos por unidad y costo de mano de obra de terramozas

\section{Correlaciones no significativas, siendo $p>\alpha=0,05$}

Costo por unidad de transporte y costo de mano de obra de choferes por unidad

Costo de materiales por vehículo y costo de mano de obra de choferes por semana

Costo de materiales por vehículo y costo de mano de obra de choferes por unidad

Elaboración del costo de la mano de obra y costo de mano de obra de choferes por semana

Elaboración del costo de mano de obra y costo de mano de obra de choferes por unidad

Costos indirectos por unidad y costos de mano de obra de choferes por semana

Costos indirectos por unidad y costos de mano de obra de choferes por unidad

\section{Trabajadores}

En relación a las actitudes de los trabajadores con respecto al control del costo de los materiales al implementarse el sistema de costos por órdenes especificas el $54,00 \%$ respondieron: definitivamente si, así mismo se observó que el $42 \%$ de los trabajadores manifiestan: probablemente si con la elaboración de los costos, mientras que el 0,0\% manifiestan: probablemente no, por otro lado el $54 \%$ de los trabajadores responden definitivamente si, en cuanto a los informes de costos, mientras que el $0 \%$ manifiestan definitivamente no.

\section{Consolidado}

Al relacionar las variables implementación del sistema y el sistema de costos por órdenes específicas de la empresa de transportes San Martin de Porres S.A del distrito de Huacho, los trabajadores opinaron definitivamente si $54 \%$, y que es importante el sistema de costos por órdenes específicas, el, $25 \%$ manifiestan probablemente si, el $21 \%$ manifiestan No estoy seguro, mientras que el $0 \%$ manifiestan probablemente no y el $0 \%$;manifestaron probablemente no así mismo con respecto a la variable sistema de costos por órdenes especificas el $58 \%$ de los trabajadores manifiestan definitivamente si, el $17 \%$ probablemente si, con un $25 \%$ No estoy seguro el $0 \%$ probablemente No, y el $0 \%$ definitivamente no.

Por lo que el $79 \%$ de los trabajadores están de acuerdo en la implementación del sistema de costos; así mismo el $75 \%$ están de acuerdo que el sistema sea de costos por órdenes específicas.

En cuanto a la prueba de hipótesis general para indicar las relaciones de sus variables se ejecutó mediante el valor $\alpha=5 \%$, para lo cual requirió de las siguientes interpretaciones: Si valor $p \geq 0,05$, se acepta hipótesis nula (Ho); si $p<0.05$, se acepta hipótesis de investigación o hipótesis alternativa $(\mathrm{Ha})$. El estadístico de prueba fue la correlación de Pearson, dado que este estadístico es apropiado para ver relaciones entre variables, obteniéndose los siguientes resultados:

Tabla 3. Correlaciones de variables

\begin{tabular}{|c|c|c|c|}
\hline & & $\begin{array}{c}\text { Variable } \\
\text { Independiente } \\
\text { Implementación } \\
\text { del Sistema }\end{array}$ & $\begin{array}{c}\text { Variable } \\
\text { Dependiente } \\
\text { Sistema de } \\
\text { Costos por } \\
\text { Órdenes } \\
\text { especificas }\end{array}$ \\
\hline $\begin{array}{l}\text { Variable } \\
\text { Independiente } \\
\text { Implementación del } \\
\text { sistema de costos }\end{array}$ & $\begin{array}{l}\text { Coeficiente de } \\
\text { correlación Rho } \\
\text { de Spearman } \\
\text { Sig. (bilateral) } \\
\text { N }\end{array}$ & 23 & $\begin{array}{r}.000 \\
23\end{array}$ \\
\hline $\begin{array}{l}\text { Variable } \\
\text { Dependiente } \\
\text { Sistema de Costos } \\
\text { por Órdenes } \\
\text { especificas }\end{array}$ & $\begin{array}{l}\text { Coeficiente de } \\
\text { correlación Rho } \\
\text { de Spearman } \\
\text { Sig. (bilateral) } \\
\text { N }\end{array}$ & $\begin{array}{r}.000 \\
23\end{array}$ & 23 \\
\hline
\end{tabular}

${ }^{* *}$. La correlación es significativa en el nivel 0,01

Fuente: Elaboración propia basado en cuestionario $(n=23)$.

El Valor de $p=0.000$, R de Spearman $=0,540$.

Con una probabilidad de error del $0.000 \%$, menor que 0.05 , existe una correlación positiva moderada y significativa entre las variables Sistema competencias docentes y procesos de acreditación. 


\section{DISCUSIÓN}

La opinión de los trabajadores respecto a la implementación del sistema de costos y el sistema de costos por órdenes específicas analizadas pone en evidencia los siguientes aspectos.

La implementación del sistema de costos influyen directamente en el sistema de costos por órdenes específicas, como señala E. Suscely, B. Paiva, N. Milagro, M. Gonzales, M. Fernando, U. Cárdenas (2010) que es necesario la aplicación de un sistema de costos por órdenes específicas para mejorar la rentabilidad den la empresa Vista Alegre S.R.L.

Asimismo para M. de José Fornos, (2003). Indica "Es el método mediante el cual se asignan todos los costos incurridos para transformar materias primas en productos terminado, asociados a un lote o pedido específico de producción". Los Costos por Órdenes de Producción son un sistema mediante el cual se acumulan los costos sobre un lote de productos similares identificados con una determinada orden de Producción. Este sistema se aplica a las industrias de Construcción, muebles, impresión, equipos y a cualquier empresa que trabaje en base a pedidos.

Se debe tener en cuenta que las empresas en general y aquellas cuyos ingresos son mayores a 1,700 UIT, están obligadas a llevar contabilidad de costos, para determinar el costo de sus inventarios y determinar las rentabilidades, no cumplir esta obligación constituye una infracción tributaria sujeta a multas que perjudicarían las rentabilidades de la empresa y los dividendos de los socios.

Una vez obtenidos y analizados los resultados de las investigaciones, nos permite aceptar la hipótesis general, es decir existe relación directa entre la implementación del sistema de costos y el sistema de costos por órdenes específicas de la empresa de transporte San Martin de Porres S.A del distrito de Huacho.

Con relación a la hipótesis especifica uno, se desprende del análisis de correlación entre las variables, Control de costos y el sistema de costos por órdenes específicas de la empresa de transporte San Martin de Porres S.A del distrito de Huacho, hay una relación, ya que al aplicarse la prueba de hipótesis se obtuvo el resultado de $\alpha=: 0.00$ que es menor a $\alpha$ (0.05).

Con respecto a la segunda hipótesis se afirma que existe una probabilidad en que la elaboración de costos influye directamente en el sistema de costos por órdenes especificas de la empresa de transporte San Martin de Porres S.A, del distrito de Huacho, según los datos de significancia $(0,000)$ obtenidos

Con relación a la tercera hipótesis se acepta porque los informes de costos influyen positivamente en el sistema de costos por órdenes específicas en la empresa de transporte San Martin de Porres S.A, del distrito de Huacho.

\section{CONCLUSIÓN}

Podemos decir que el sistema de costos es un procedimiento para calcular los costos en cualquier tipo de empresas, industriales, comerciales o de servicio.

Que el sistema que deben aplicar las empresas de transporte es el sistema de costos por órdenes específicas, porque permite controlar los costos por cada unidad de transporte con mayor seguridad

El sistema de costos por órdenes especificas controlan los costos de los materiales, los costos de la mano de obra y los costos indirectos, asimismo determinan las rentabilidades con más seguridad

La implementación del sistema de costos, causaría un gran impacto en la sociedad empresarial del transporte, porque les permitirá una mejora continua en sus costos y decisiones en renovación de flota, medir sus financiamientos, y captación de capitales ajenos.

Si tenemos en cuenta que no existe un marco de referencia bajo el cual se desarrollen políticas adecuadas de reducción de costos en el servicio, o mejorar las inversiones de la empresa para que estas permitan asegurar la calidad en el servicio prestado al cliente final, lo que se ha traducido en la principal problemática en las empresas de este gremio.

Sin embargo existen modelos diseñados para optimizar los costos tales como el ABC, el sistema de costos estándar etc, para calcular el costo de servicios, estos tienen una gran complejidad en el desarrollo de los mismos, además de su costosa y larga implementación, por esta razón nuestra investigación encuentra a la luz del sistema de ordenes específicas, un sistema de costeo más sencillo para la empresa debido a que su desarrollo no es costoso y es de rápida implementación, lo cual tendría mayores ventajas frente al contexto de funcionamiento de estas empresas. 


\section{AGRADECIMIENTO}

Al personal ejecutivo, directivo y trabajadores administrativos choferes y terramozas de la empresa de transporte san Martin de Porres S.A por la atención que nos brindaron.

A los alumnos Gómez Díaz, Yola Roxana, Porles Moreyra, Elena, Rojas Bellido, Víctor y Huarac Capillo, Yoselyn que colaboraron en la realización de las encuestas y la aplicación de los cuestionarios, Al profesor estadístico de la universidad Sifuentes Damián, Anibal por su colaboración en la aplicación de las pruebas estadísticas.

\section{REFERENCIAS BIBLIOGRÁFICAS}

Bach Juan R (2001) Ediciones Bach S.R.L Argentina.

Bazalar Paiva, Evelig Susely-Morales Nathaly Del Milagrto (2010) Aplicación de un sistema de costos por órdenes específicas para mejorar la rentabilidad de la empresa Vista Alegre SRL.

Becker Morton, Lule Jacobsen y David Jiménez (1988) Contabilidad de Cotos, Un Enfoque Administrativo y De Gerencia. Ed. McGraw-Hill.

Brito José A.. (2011) Contabilidad Básica e Intermedia Ediciones Centro de Contadores 6ta Edición .

Erick Kolher( 1994) Contabilidad de Costos Editorial Limusa S.A. 5ta Edición.

Fernández Ibáñez, Lima(2011) Diseño de un Sistema de Costos por Ordenes Especificas para la fabricación de Carrocerías .

Gómez Bravo, Oscar (2001) Contabilidad De Costos Editorial McGraw-Hill Bogotá Colombia.

Gonzalo Sinistrani, Polanco Luis (2008) Contabilidad del sistema de información para la organización.

Haragadon Bernard y Munera (1988) Contabilidad de los Costos.

Hidalgo Ortega Jesús (1995)Contabilidad de Costos Editorial Perú.

M. De José Fornos (2003) Contabilidad de Costos.

National Association of Accoutant.

Railph S Polimeni (1997) Contabilidad de Costos: Conceptos y aplicaciones para la toma de decisiones, Ed. McGraw Hill Bogotá Colombia.

Santa Cruz Ramos, Alfonzo (2006) Costos Teoria y Practica. Instituto de investigacion el pacifico. 